\section{Wingful, an extracellular feedback inhibitor of Wingless}

\author{
Offer Gerlitz and Konrad Basler ${ }^{1}$ \\ Institut für Molekularbiologie, Universität Zürich, \\ CH-8057 Zürich, Switzerland
}

Secreted peptide signals control many fundamental processes during animal development. Proper responses to these signals require cognate inducible feedback antagonists. Here we report the identification of a novel Drosophila Wingless (Wg) target gene, wingful (wf), and show that it encodes a potent extracellular feedback inhibitor of Wg. In contrast to the cytoplasmic protein Naked cuticle (Nkd), the only known Wg feedback antagonist, Wf functions during larval stages, when Nkd function is dispensable. We propose that $\mathrm{Wf}$ may provide feedback control for the long-range morphogen activities of Wg.

Received March 18, 2002; accepted March 28, 2002.

Pattern formation during animal development is largely controlled by intercellular signaling events. Peptide signals secreted by some cells regulate a variety of cellular processes in other cells by activating signal transduction pathways, which induce cell fate changes and morphogenetic responses. Over the past decade it has been recognized that the precision of developmental signaling depends largely on the employment of positive- and negative-feedback control (Perrimon and McMahon 1999; Freeman 2000). Negative-feedback inhibitors are widely used to spatially and temporally limit responses to developmental signals, enabling an intricate accuracy of pattern formation (Freeman 2000).

Wnt proteins are secreted signaling molecules that direct growth and cell fates in processes as diverse as embryonic segmentation, CNS patterning, and limb development (for review, see Wodarz and Nusse 1998). The protein encoded by the Drosophila segment-polarity gene wingless $(w g)$ is a defining member of the Wnt family (for review, see Nusse and Varmus 1992). In embryos, Wg acts as a short-range inducer: it is secreted by subpopulations of cells within each segment and received by their immediate neighbors (van den Heuvel et al. 1989). Later in development, $\mathrm{Wg}$ plays an essential role in the primordia of the adult appendages, where it acts directly on cells, over a range of many cell diameters, to organize their patterns of differentiation (Zecca et al. 1996; Neumann and Cohen 1997).

A negative-feedback inhibitor has recently been iden-

[Key Words: Negative feedback control; Wingless signaling; pattern formation]

${ }^{1}$ Corresponding author.

E-MAIL basler@molbio.unizh.ch; FAX 41-1-635-6864.

Article and publication are at http://www.genesdev.org/cgi/doi/10.1101/ gad.991802. tified that limits the effects of the Wg signal (Zeng et al. 2000). The naked cuticle (nkd) gene encodes an EF hand protein that regulates embryonic $\mathrm{Wg}$ activity by acting as an inducible antagonist of the Wg transduction component Dishevelled (Rousset et al. 2001). Unexpectedly, the $n k d$ product plays no discernible role at later stages of development, such as during the patterning of imaginal discs (Zeng et al. 2000). This is particularly surprising because the spatial patterning activities of the Wg morphogen are especially demanding during these processes (Zecca et al. 1996; Neumann and Cohen 1997; Strigini and Cohen 2000). It is tempting to suggest, therefore, that another feedback inhibitor is used to antagonize Wg activity in larval tissues.

Most, if not all, genes encoding negative-feedback inhibitors are transcriptionally induced in all cells transducing the respective signal, with no apparent stage or tissue specificity. This behavior contrasts that of the ef-
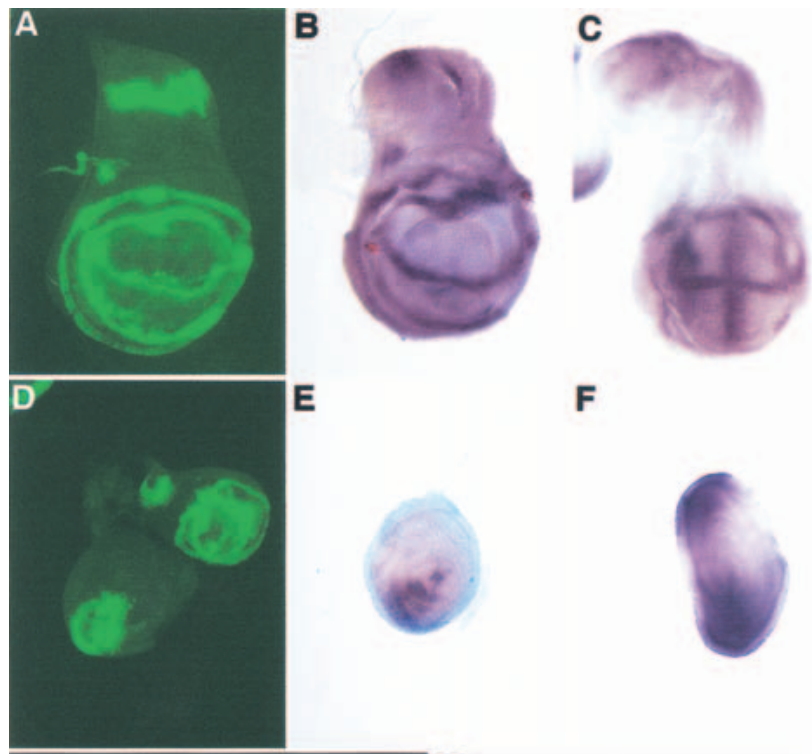

E

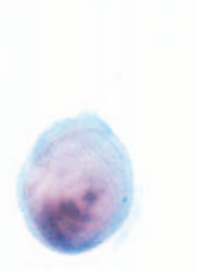

F
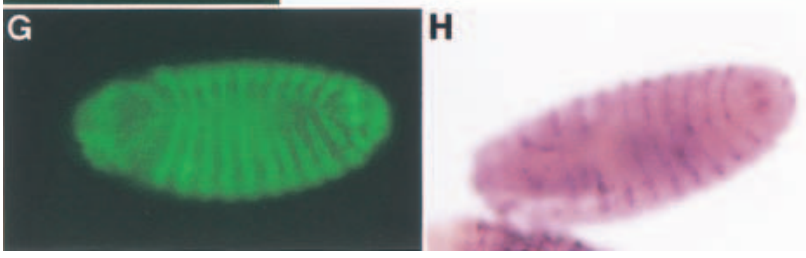

Figure 1. Expression pattern of $w f$, a target of $\mathrm{Wg}$ signaling $(A-C)$ Wing imaginal discs (anterior to the left, dorsal up), showing the activity of the Gal4 enhancer trap insertion P[S145] revealed by GFP fluorescence $(A)$, or in situ hybridizations of a wild-type $(B)$ and a $d p p-G a 14 U A S-w g$ disc $(C)$ with an RNA probe derived from CG13076. Note the ectopic expression of wf in a stripe along the anteroposterior compartment boundary in C. $(D-F)$ Leg imaginal discs, showing the expression of $P$ [S145] $(D)$ or of CG13076 transcripts in wild-type $(E)$ or $d p p-G a 14$ $U A S-w g$ animals $(F)$. Note the ectopic expression of $w f$ in dorsal regions of the leg disc. In $D$, a haltere disc is visible to the upper right of the leg disc. $(G, H)$ Dorsal view (anterior to the right) of embryos showing the wf expression pattern visualized by $P[S 145]$ activity $(G)$ or in situ hybridization $(H)$. 
A

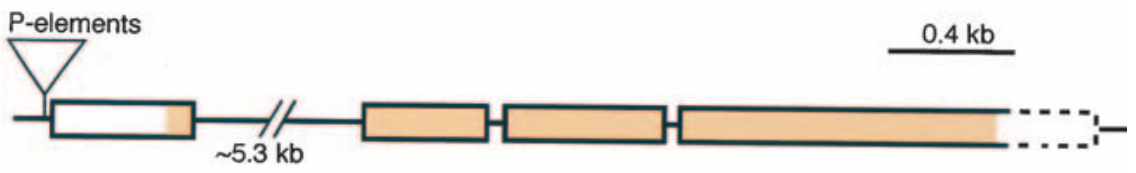

B

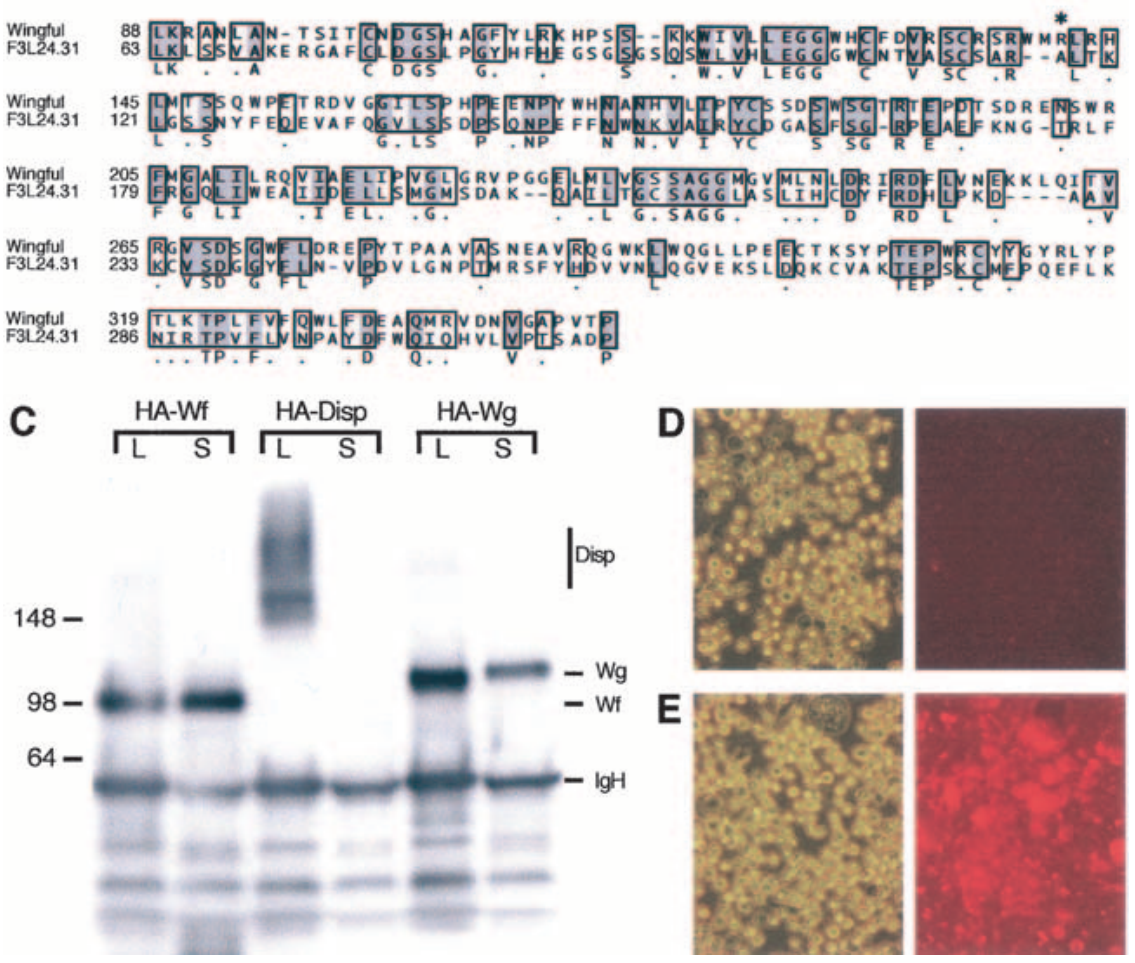

Figure 2. Wf encodes a secreted protein with homology to pectin acetylesterases. $(A)$ Structural organization of the wf locus, indicating the position of the Gal4 P-element insertions. Boxes represent exons, coding sequences are colored. (B) Alignment of the Wf protein sequence with the putative pectin acetylesterase family member F3L24.31 from Arabidopsis thaliana (on chromosome III BAC F3L24, GenBank accession no. AC011436). The position of the stop codon present in allele $w f^{141}$ is indicated by an asterisk. The complete wf mRNA and protein sequence has been deposited to GenBank (accession no. AY078993). (C) Western blot of an immunoprecipitation experiment using supernatants (S) and cell lysates (L) from S2 cells transfected with HA-tagged wf (HA-Wf), dispatched $(H A-d i s p)$, and $w g(H A-W g)$. Proteins were precipitated with a monoclonal anti-HA antibody. HA-Wf and HA-Wg are readily secreted as they are detected in lysates and supernatants. $(D)$ Supernatants of $H A$-wg-expressing cells $(D)$ and $H A$-wf-expressing cells $(E)$ have been incubated with nontransfected S2 cells. Binding to cell surfaces was monitored by an anti-HA antibody, followed by an Alexa 594 goat anti-mouse secondary antibody, and is shown to the right of the bright field images.

fector targets of these signals, the induction of which generally depends on the developmental state of the cell receiving it (Freeman 2000). For example, although the expression of the Hedgehog $(\mathrm{Hh})$ feedback antagonist Patched is up-regulated in all cells known to transduce the Hh signal (Forbes et al. 1993; Goodrich et al. 1996), one of the key effector targets of the Hh signal in discs, decapentaplegic $(d p p)$, is only induced in larval but not embryonic cells (Basler and Struhl 1994; Tabata and Kornberg 1994). Similarly, the induction of the Smad feedback inhibitor Dad is a universal response to Dpp signaling (Tsuneizumi et al. 1997), whereas nonfeedback targets of Dpp, such as pannier, tinman, labial, and op- tomoter-blind, are expressed in different subsets of Dpp-induced cells (for review, see Affolter et al. 2001).

Here we set out to identify a larval Wg feedback antagonist on the basis of its putative expression pattern. By screening a large collection of enhancer trap lines, we isolated a panWg-target, wingful (wf); we show that it encodes a secreted protein with sequence homology to hydrolytic enzymes. A loss-of-function mutation in $w f$ results in phenotypes reminiscent of $\mathrm{Wg}$ gain-of-function situations, whereas overexpression of $\mathrm{Wf}$ severely inhibits Wg signaling activity. Thus Wf functions, indeed, as a potent feedback inhibitor of $\mathrm{Wg}$ and complements the embryonic nkd system both temporally and mechanistically by shaping the larval response gradient to $\mathrm{Wg}$ morphogen signaling.

\section{Results and Discussion}

Identification of wingful as a Wg target gene

A library of 2000 Gal4 enhancer trap $P$-element insertions was established, each of which reports a gene expression pattern in the wing imaginal disc (Gerlitz et al. 2002). This collection was screened with a UAS-GFP reporter for lines that show a $w g$-like expression pattern. There were 11 insertions identified that reported $w g$ like gene expression in the embryonic epidermis and all imaginal discs (Fig. 1A,D,G). Four of these lines (S180, ND382, S476, S554) contained an insertion in the $w g$ gene itself (data not shown); the other seven lines (S141, S145, S163, S330, ND337, ND339, ND634) all carried a $P$-element insertion at cytological position 72D, only a few base pairs upstream of gene CG13076, referred to as wingful (wf). These enhancer trap insertions indeed report the expression of $w f$, as revealed by RNA in situ hybridization (Fig. 1B,E,H). wf is ectopically expressed upon $\mathrm{Wg}$ misexpression (Fig. 1C,F), indicating that $w f$ is a Wg target throughout larval development.

wf encodes a secreted protein with structural homology to pectin acetylesterases

RACE analysis revealed that the first exon of $w f$ is incorrectly predicted in the Drosophila genome annotations (http://flybase.bio.indiana.edu) and instead is located $5.3 \mathrm{~kb}$ further upstream (Fig. 2A). The resultant open reading frame of $w f$ codes for a presumptive protein of 671 amino acids, with an N-terminally situated signal 
sequence. The wf product is readily secreted from transfected Drosophila cells (Fig. 2C) and has a noticeable propensity to adhere to the surfaces of intact cells (Fig. 2D,E). The analysis of the Wf protein sequence reveals a significant structural homology to a subfamily of poorly characterized hydrolases related to plant pectin acetylesterase (Fig. 2B; Breton et al. 1996). Together, these results suggest that the product of the off gene may catalyze the hydrolytic cleavage of an extracellular substrate.

\section{Loss of Wf function causes a gain of Wg activity}

To test our hypothesis that the panWg-target wf encodes an inhibitor of Wg activity, we sought to abolish wf function by genetic means. From a collection of six EMS-induced lethal mutations, located between the distal breakpoint of $D f(3 L) s t-f 13$ and the proximal breakpoint of $D f(3 L)$ brm 11 (Meléndez et al. 1995), we identified a putative null allele of $w f$ with a stop codon at amino acid position 141, encoding a severely truncated protein. Animals homozygous for $w f^{141}$ or animals of the genotype $w f^{141} /$ $D f 3(3 L)$ st- $f 13$ die during pupal stages, and show various phenotypes. The most prominent of these are patterning defects in the wing imaginal disc.

Wg signaling plays at least two distinct roles during wing development. Early reduction of $w g$ activity results in a wing-to-notum transformation, indicating a requirement for Wg in defining the wing blade primordium (Morata and Lawrence 1977; Ng et al. 1996), but later reductions cause the loss of wing margin and adjacent tissue, indicating its subsequent role in specifying the wing margin and organizing wing blade development (Couso et al. 1994; Diaz-Benjumea and Cohen 1995; Rulifson and Blair 1995). As shown in Figure 3, wf animals show phenotypes opposite to both classes. Wing discs mutant for wf are enlarged with an extended wing blade region (hence the name wingful). Often these discs contain two wing pouches at the expense of notal structures (Fig. 3B). Although we did not detect an apparent expansion of Distalless-1acZ (Dll-lacZ) expression along the dorsoventral axis, there was a significant increase in the number of cells expressing neuralized (Fig. 3C), a high-threshold Wg target expressed in neural wing margin cells (Zecca et al. 1996). Consistent with this observation, rare adult escapers mutant for wf show a dramatically increased number of mechanosensory bristles in the wing (Fig. 3J). Wg signaling also distinguishes between sternite and ventral pleura development in the adult abdomen (Shirras and Couso 1996). wf adult escapers show extra sternite bristles (Fig. 3F), an effect that was also observed with ectopic expression of wg (Shirras and Couso 1996). Finally, wf adults show extra dorsocentral bristles (Fig. $3 \mathrm{H})$, sensory organs on the notum whose specification has been shown to depend on $w g$ activity (Phillips and
Whittle 1993; Calleja et al. 1996). Taken together, these results show that the absence of off function causes a gain of $\mathrm{Wg}$ activity in developing adult tissues. Therefore, the function of the wild-type $w f$ product is to limit Wg signaling activity.

\section{Gain of Wf function causes a loss of Wg signaling}

A further prediction of our assumption that Wf functions as a Wg feedback inhibitor is that wf overexpression should lead to $w g$ loss-of-function phenotypes. We present three lines of evidence to show that this is, indeed, the case. First, we replaced one of the $w f$ enhancer trap $P$-element insertions with an EP element positioning 10 $U A S$ sites upstream of the wf gene, rendering it transcriptionally responsive to Gal4 expression. Alterations of $w f$ expression had unusually potent effects, as all commonly used Gal4 drivers caused lethality in combination with UAS-wf. The only exceptions were S168-Gal4 and scalloped-Gal4, which are expressed in the wing pouch and represent Wg targets (Fig. 4A,C), providing a selfregulating circuit in combination with $U A S-W f$ (see below). Adult animals carrying the S168-Gal4 and UAS-Wf transgenes have severely reduced wings that lack all wing margin structures (Fig. 4E,F). Second, we analyzed the expression of two target genes in this context. S168Gal4 expression was virtually abolished by UAS-Wf expression (Fig. 4A,B), whereas the expression domain of $w g$ itself is expanded. It had previously been reported that Wg narrows its own domain of expression, because 

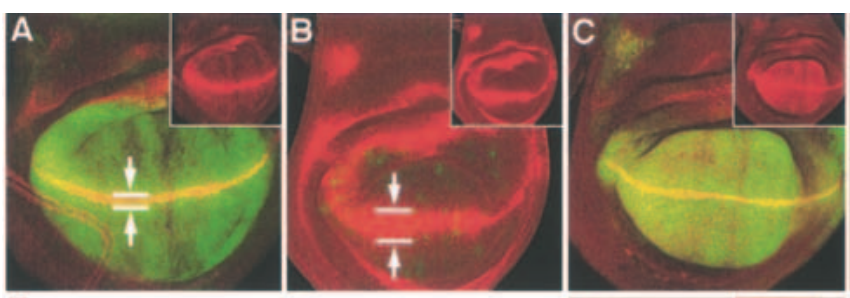

E
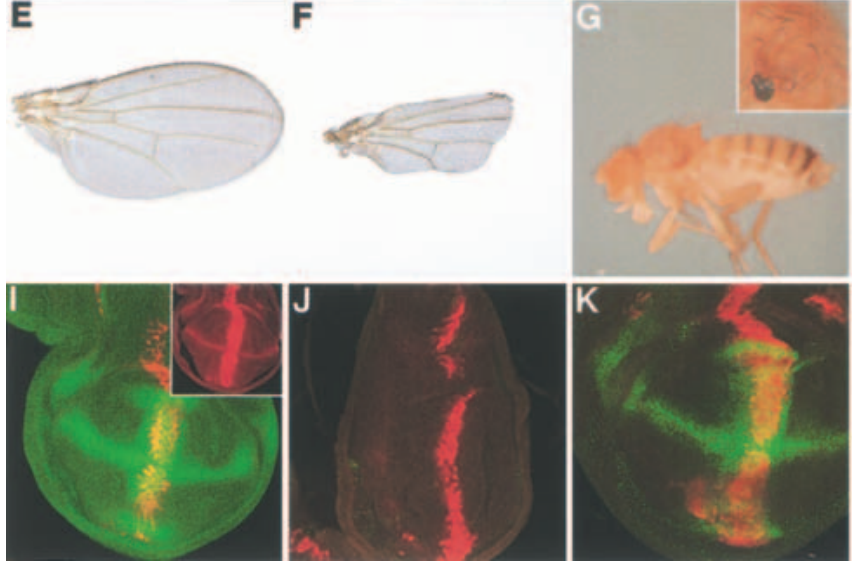

Figure 4. wf gain-of-function phenotypes. $(A, B)$ Expression of S168-Gal4 visualized with $U A S-G F P$ (in green) and Wg protein (red) in the absence $(A)$ and presence $(B)$ of the $U A S-w f$ transgene. Note the reduction of $S 168$ expression and widening of the wg expression domain along the dorso-ventral boundary in the presence of higher Wf levels (see arrows). $(C, D)$ Expression of scalloped-Gal4 visualized with UAS-GFP (in green) and $\mathrm{Wg}$ protein $(\mathrm{red})$ in the absence $(C)$ and presence $(D)$ of a $U A S-w f$ transgene. Note the absence of scalloped and $w g$ pouch expression owing to the wing-to-notum phenotype induced by overexpression of Wf. Insets in $A-C$ show low-magnification images of the Wg staining. $(E, F)$ Adult wings of wild-type $(E)$ and S168-Gal4 UAS-Wf adults $(F) .(G)$ Wing-to-notum transformation of a scalloped-Gal4 UAS-wf adult. A higher magnification of the extra notum is shown in the inset. $(H)$ Wing imaginal disc expressing UAS-Wf under the control of $d p p-G a 14$ (green), resulting in a wing-to-notum transformation and a concomitant symmetrical expression of $\mathrm{Wg}($ red). $(I, J)$ Dll-lacZ expression (green) in wing discs expressing $U A S-W g$ under $d p p-G a l 4$ control in the absence $(I)$ or presence $(J)$ of the UAS-Wf transgene. Wg expression is visualized in red. $(K, L)$ Dll-lacZ expression (green) in wing discs expressing UAS-NRT-wg under $d p p-G a l 4$ control in the absence $(K)$ or presence $(L)$ of the $U A S-W f$ transgene. Wg expression is visualized in red. Note the elimination of $N R T-w g$-induced Dll-lacZ expression (green) by ectopic Wf.

a reduction in $\mathrm{Wg}$ signal transduction causes ectopic $w g$ transcription (Rulifson et al. 1996). Finally, and perhaps most strikingly, driving expression of wf with scallopedGal4 results in a wing-to-notum transformation (Fig. $4 \mathrm{D}, \mathrm{G})$, the founding loss-of-function phenotype of the wg gene (Sharma and Chopra 1976).

\section{Wf blocks the signaling activities of free and tethered $W g$}

Based on its structural features as a secreted protein with homologies to pectin acetylesterases, Wf could exert its function by modifying polysaccharide-based properties of cell surface proteins and thereby impeding the intercellular movement of the Wg protein. Alternatively, Wf could counteract Wg signaling by modifying the transducing properties of $\mathrm{Wg}$ or one of its receptors. To distinguish between these two possibilities, we tested whether Wf also antagonizes a derivative of $\mathrm{Wg}$, Nrt-Wg, that is tethered to the cell surface and does not move
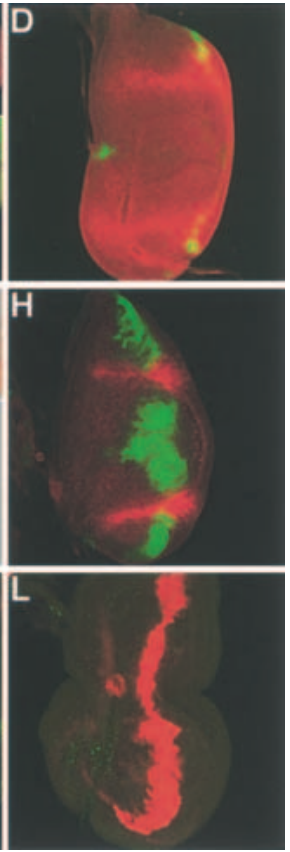

through tissue (Zecca et al. 1996). Expression of Wg or Nrt-Wg driven by $d p p-G a l 4$ results in a robust activation of ectopic Dll-lacZ expression (Fig. 4I,K; Zecca et al. 1996). Surprisingly, wf expression extinguishes Dll-lacZ expression induced by tethered $\mathrm{Wg}$, as well as that induced by free $\mathrm{Wg}$ (Fig. 4J,L). From this experiment it can be ruled out that the primary function of $\mathrm{Wf}$ is to impede the extracellular transport of $\mathrm{Wg}$. Therefore, Wf must interfere with the signaling activities of either $\mathrm{Wg}$ or its receptor components. Because we were unable to detect a physical interaction between $\mathrm{Wf}$ and $\mathrm{Wg}$, Frizzled-2 (Dfz2), and its LRP-like partner Arrow in tissue culture systems (data not shown), we favor the view that Wf inhibits the activity of a coreceptor component, such as Dally or Dally-like (Dly), proteoglycans that appear to participate in Wg reception (Lin and Perrimon 1999; Tsuda et al. 1999; Baeg et al. 2001). Wf may inhibit such receptor components via its presumptive esterase activity, for example, by modifying Dally or Dly glycosaminoglycan chains. A definitive proof for this mode of action could be achieved by the genetic demonstration that in larval dally dly double-mutant situations, loss of wf function has no antagonistic effect.

\section{Temporal and mechanistic differences between feedback inhibitors of $\mathrm{Wg}$}

The discovery of Wingful as an essential Wg feedback antagonist may provide an explanation of why Nkd has no apparent role in imaginal tissues of Drosophila. The function of Nkd may be superseded by that of $\mathrm{Wf}$, which functions in a powerful negative-feedback loop in adult development. Conversely, when we removed both maternal and zygotic components of $w f$, no obvious requirement was observed for Wf in embryonic development, possibly because the $n k d$ system is operative at this stage of development. Both Naked and Wf can, however, inhibit Wg signaling throughout development if they are overexpressed, but each of them is operating more effectively at only one of the two stages. It may not be coincidence that Nkd as the intracellular feedback antagonist is used during embryonic patterning, where $\mathrm{Wg}$ functions at short range, whereas $\mathrm{Wf}$ as a secreted extracellular antagonist primarily regulates patterning processes that depend on long-range Wg signaling. Wf functions nonautonomously (Fig. 3K) and, like Argos, a secreted feedback antagonist of the Drosophila EGF system (Golembo et al. 1996), may have a different range of action compared with the primary signal, providing an intricate means to shape the range and slope of the cellular responses to a morphogen gradient. 


\section{Materials and methods}

Drosophila stocks and genetics

The establishment of the Gal4 enhancer trap collection is described in Gerlitz et al. (2002). P-element $P$ [S145], which maps 8 bp upstream of the $5^{\prime}$ end of the $w f$ transcription unit, was replaced with the $y^{+}$-marked EP element DA530inv (D. Nellen and K. Basler, unpubl.) essentially as described by Sepp and Auld (1999). Experimental larvae for dissection were identified by the absence of the Tubby genotype present on the balancer chromosome TM6b. Dll-lacZ, neur-lacZ, UAS-HA-Wg, and UAS-HA$N r t-w g$ are described in Zecca et al. (1996).

Immunoblotting and histochemistry

S2 cells were transfected with UAS constructs in combination with a tubulina1-Gal4 plasmid. Cell lysates and supernatants were incubated with anti-HA antibody; immunocomplexes were resolved by SDS-PAGE, followed by blotting with anti-HA antibodies. Cell surface staining was assayed as follows: S2 cells were incubated with supernatant of $H A-W f-$ or HA-Wg-expressing cells at room temperature for $2 \mathrm{~h}$, washed with serum-free medium, and stained with mouse monoclonal $\alpha$-HA and polyclonal goat Alexa 594 secondary antibodies. Imaginal discs from third instar larvae were fixed and stained by standard techniques with mouse monoclonal $\alpha-\mathrm{Wg}$ (gift from S. Cohen, EMBL, Heidelberg, Germany) and polyclonal $\alpha-\beta \mathrm{Gal}$ (Cappel); mouse monoclonal $\alpha-\mathrm{HA}$ (BAbCO), and Alexa 488 and 594 fluorescent secondary antibodies (Molecular Probes).

\section{Acknowledgments}

We thank S. DiNardo for the arrow cDNA, Z. Chamoun for help with confocal microscopy and figures, E. Frei for RACE analysis, and members of the lab for comments on the manuscript. This project was supported by the Swiss National Science Foundation and the Kanton of Zürich.

The publication costs of this article were defrayed in part by payment of page charges. This article must therefore be hereby marked "advertisement" in accordance with 18 USC section 1734 solely to indicate this fact.

\section{References}

Affolter, M., Marty, T., Vigano, M.A., and Jazwinska, A. 2001. Nuclear interpretation of Dpp signaling in Drosophila. EMBO J. 20: 32983305.

Baeg, G.H., Lin, X., Khare, N., Baumgartner, S., and Perrimon, N. 2001. Heparan sulfate proteoglycans are critical for the organization of the extracellular distribution of Wingless. Development 128: 87-94.

Basler, K. and Struhl, G. 1994. Compartment boundaries and the control of Drosophila limb pattern by hedgehog protein. Nature 368: 208214.

Breton, C., Bordenave, M., Richard, L., Pernollet, J.C., Huet, J.C., Perez, S., and Goldberg, R. 1996. PCR cloning and expression analysis of a cDNA encoding a pectinacetylesterase from Vigna radiata L. FEBS Lett. 388: 139-142.

Calleja, M., Moreno, E., Pelaz, S., and Morata, G. 1996. Visualization of gene expression in living adult Drosophila. Science 274: 252-255.

Couso, J.P., Bishop, S.A., and Martinez Arias, A. 1994. The wingless signalling pathway and the patterning of the wing margin in Drosophila. Development 120: 621-636.

Diaz-Benjumea, F.J. and Cohen, S.M. 1995. Serrate signals through Notch to establish a Wingless-dependent organizer at the dorsal/ventral compartment boundary of the Drosophila wing. Development 121: $4215-4225$.

Forbes, A.J., Nakano, Y., Taylor, A.M., and Ingham, P.W. 1993. Genetic analysis of hedgehog signaling in the Drosophila embryo. Development Suppl.: 115-124.

Freeman, M. 2000. Feedback control of intercellular signalling in development. Nature 408: 313-319.

Gerlitz, O., Nellen, D., Ottiger, M., and Basler K. 2002. A screen for genes expressed in Drosophila imaginal discs. Int. J. Dev. Biol. 46: 173-176.

Golembo, M., Schweitzer, R., Freeman, M., and Shilo, B.-Z. 1996. argos transcription is induced by the Drosophila EGF receptor pathway to form an inhibitory feedback loop. Development 122: 223-230.

Goodrich, L.V., Johnson, R.L., Milenkovic, L., McMahon, J.A., and Scott, M.P. 1996. Conservation of the hedgehog/patched signaling pathway from flies to mice: Induction of a mouse patched gene by Hedgehog. Genes \& Dev. 10: 301-312.

Lin, X. and Perrimon, N. 1999. Dally cooperates with Drosophila Frizzled 2 to transduce Wingless signalling. Nature 400: 281-284.

Meléndez, A., Li, W., and Kalderon, D. 1995. Activity, expression and function of a second Drosophila Protein Kinase A catalytic subunit gene. Genetics 141: 1507-1520.

Morata G. and Lawrence P.A. 1977. The development of wingless, a homeotic mutation of Drosophila. Dev. Biol. 56: 227-240.

Neumann, C.J. and Cohen, S.M. 1997. Long-range action of Wingless organizes the dorsal-ventral axis of the Drosophila wing. Development 124: 871-880.

Ng, M., Diaz-Benjumea, F.J., Vincent, J.P., Wu, J., and Cohen, S.M. 1996. Specification of the wing by localized expression of wingless protein Nature 381: 316-318.

Nusse, R. and Varmus, H.E. 1992. Wnt genes. Cell 69: 1073-1087.

Perrimon, N. and McMahon, A.P. 1999. Negative feedback mechanisms and their roles during pattern formation. Cell 97: 13-16.

Phillips, R.G. and Whittle, J.R.S. 1993. Wingless expression mediates determination of peripheral nervous system elements in late stages of Drosophila wing disc development. Development 118: 427-438.

Rousset, R., Mack, J.A., Wharton, K.A., Axelrod, J.D., Cadigan, K.M., Fish, M.P., Nusse, R., and Scott, M.P. 2001. Naked cuticle targets disheveled to antagonize Wnt signal transduction. Genes \& Dev. 15: 658-671.

Rulifson, E.J. and Blair, S.S. 1995. Notch regulates wingless expression and is not required for reception of the paracrine wingless signal during wing margin neurogenesis in Drosophila. Development 121: 2813-2824.

Rulifson, E.J., Micchelli, C.A., Axelrod, J.D., Perrimon, N., and Blair, S.S. 1996. wingless refines its own expression domain on the Drosophila wing margin. Nature 384: 72-74.

Sepp, K.J. and Auld, V.J. 1999. Conversion of lacZ enhancer trap lines to GAL4 lines using targeted transposition in Drosophila melanogaster. Genetics 151: 1093-1101

Sharma, R.P. and Chopra, V.L. 1976. Effect of the Wingless (wg1) mutation on wing and haltere development in Drosophila melanogaster. Dev. Biol. 48: 461-465.

Shirras, A.D. and Couso, J.P. 1996. Cell fates in the adult abdomen of Drosophila are determined by wingless during pupal development. Dev. Biol. 175: 24-36.

Strigini, M. and Cohen, S.M. 2000. Wingless gradient formation in the Drosophila wing. Curr. Biol. 10: 293-300.

Tabata, T. and Kornberg, T.B. 1994. Hedgehog is a signaling protein with a key role in patterning Drosophila imaginal discs. Cell 76: 89-102.

Tsuda, M., Kamimura, K., Nakato, H., Archer, M., Staatz, W., Fox, B., Humphrey, M., Olson, S., Futch, T., Kaluza, V., et al. 1999. The cell-surface proteoglycan Dally regulates Wingless signalling in Drosophila. Nature 400: 276-280.

Tsuneizumi, K., Nakayama, T., Kamoshida, Y., Kornberg, T.B., Christian, J.L., and Tabata, T. 1997. Daughters against dpp modulates dpp organizing activity in Drosophila wing development. Nature 389: 627-631.

van den Heuvel, M., Nusse, R., Johnston, P., and Lawrence, P.A. 1989. Distribution of the wingless gene product in Drosophila embryos: A protein involved in cell-cell communication. Cell 59: 739-749.

Wodarz, A. and Nusse, R. 1998. Mechanisms of Wnt signaling in development. Annu. Rev. Cell Dev. Biol. 14: 59-88.

Zecca, M., Basler, K., and Struhl, G. 1996. Direct and long-range action of a wingless morphogen gradient. Cell 87: 833-844.

Zeng, W., Wharton, K.A., Mack, J.A., Wang, K., Gadbaw, M., Suyama, K., Klein. P.S., and Scott, M.P. 2000. Naked cuticle encodes an inducible antagonist of Wnt signaling. Nature 403: 789-795. 


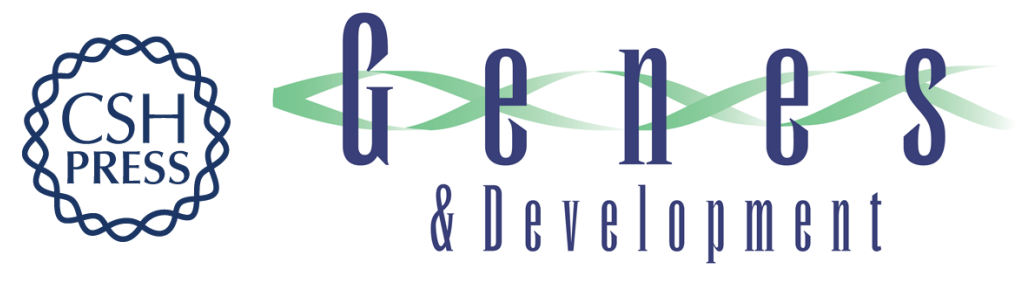

\section{Wingful, an extracellular feedback inhibitor of Wingless}

Offer Gerlitz and Konrad Basler

Genes Dev. 2002, 16:

Access the most recent version at doi:10.1101/gad.991802

\section{Related Content}

References

\section{License}
Email Alerting
Service

A Wingful of Wingless

Sci. STKE May , 2002 2002: tw179-TW179

This article cites 33 articles, 13 of which can be accessed free at: http://genesdev.cshlp.org/content/16/9/1055.full.html\#ref-list-1

Articles cited in:

http://genesdev.cshlp.org/content/16/9/1055.full.html\#related-urls

Receive free email alerts when new articles cite this article - sign up in the box at the top right corner of the article or click here.

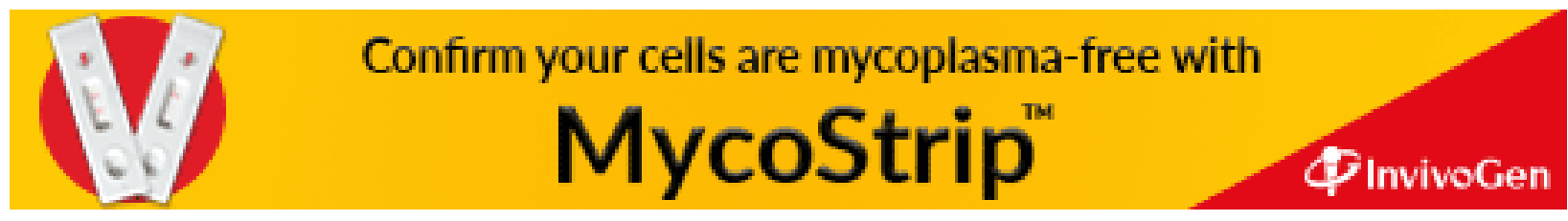

